\title{
Learning cross-lingual phonological and orthographic adaptations: a case study in improving neural machine translation between low-resource languages
}

\author{
Saurav Jha ${ }^{1}$, Akhilesh Sudhakar ${ }^{2}$, and Anil Kumar Singh ${ }^{2}$ \\ ${ }^{1}$ MNNIT Allahabad, Prayagraj, India \\ ${ }^{2}$ IIT (BHU), Varanasi, India
}

\begin{abstract}
ABSTRAGT
Out-of-vocabulary (OOV) words can pose serious challenges for machine translation (MT) tasks, and in particular, for low-resource language (LRL) pairs, i.e., language pairs for which few or no parallel corpora exist. Our work adapts variants of seq2seq models to perform transduction of such words from Hindi to Bhojpuri (an LRL instance), learning from a set of cognate pairs built from a bilingual dictionary of Hindi-Bhojpuri words. We demonstrate that our models can be effectively used for language pairs that have limited parallel corpora; our models work at the character level to grasp phonetic and orthographic similarities across multiple types of word adaptations, whether synchronic or diachronic, loan words or cognates. We describe the training aspects of several character level NMT systems that we adapted to this task and characterize their typical errors. Our method improves BLEU score by 6.3 on the Hindi-to-Bhojpuri translation task. Further, we show that such transductions can generalize well to other languages by applying it successfully to Hindi-Bangla cognate pairs. Our work can be seen as an important step in the process of: (i) resolving the OOV words problem arising in MT tasks; (ii) creating effective parallel corpora for resource constrained languages; and (iii) leveraging the enhanced semantic knowledge captured by wordlevel embeddings to perform character-level tasks.
\end{abstract}

Keywords: neural machine translation, Hindi, Bhojpuri, word transduction, low resource language, attention model 
With recent advances in the field of machine translation (MT) - and in neural machine translation (NMT) in particular - there has been an increasing need to shift focus to out-of-vocabulary (OOV) words (Wu et al. 2016; Sennrich et al. 2016). In the case of low resource languages (LRLs), which lack linguistic resources such as parallel corpora, most words are OOV words; this is problematic. Current dataintensive translation systems work poorly with OOV words for such languages, purely because of a severe lack of resources. Hence, for such languages, it becomes necessary to deal with OOV words in specific ways, outside the ambit of general-purpose NMT systems. Even in the case of resource-rich languages, methods to deal with OOV words are still being actively researched (Pham et al. 2018; Luong and Manning 2016).

Many approaches (as elaborated upon in Section 2) have been investigated to deal with the OOV problem. In this paper, we use the method of 'transduction', learned from a dictionary of cognate word pairs from Hindi and Bhojpuri. The fundamental guiding principle of our approach is the fact that Bhojpuri and Hindi are closely related languages, and hence have a good amount of vocabulary overlap, while sharing orthographic and phonetic traits. These two languages have common ancestors, and both of them employ the orthographically shallow alpha-syllabic Devanagari script. Tracing the origin of a considerable portion of the modern Bhojpuri vocabulary could weakly suggest that many of Hindi words got adapted to the local Bhojpuri phonology with the passage of time. This is a well-known phenomenon and it can be observed in other pairs of closely related languages (Macedonian - Bulgarian; Spanish - Catalan; Turkish - Crimean Tatar; Czech - Slovak; Irish - Scottish Gaelic) which share a close ancestor within the language family they belong to.

The Indian linguistic space, as reported by Grierson (1928), has 179 independent languages and 544 dialects. Similarly, the survey of Mahapatra et al. (1989) demonstrates that there are at least 50 Indian languages in which writing and publishing are done in substantial quantity. However, a majority of these languages lack proper linguistic resources. Hindi - being the lingua franca of the 'Hindi belt' (most parts of the north) of India - is a commonly spoken language in more than 
10 states and has (according to one of the views ${ }^{1}$ ) seven major closely related languages, often called 'dialects' or 'sub-languages', (namely, Awadhi, Bagheli, Bhojpuri, Bundeli, Haryanvi, Kanauji and Khari Boli) (Mishra and Bali 2011). Despite it having 33 million native speakers in India and over 6 million native speakers outside India, ${ }^{2}$ Bhojpuri still suffers from the lack of language resources. So far, very little work has been done towards developing language resources (Singh and Jha 2015), resulting in scarcity of resources such as a Bhojpuri lexical database or parallel corpus that could have made state-of-theart machine translation (MT) systems accessible to this language.

Due to the lack of such resources, neither traditional phrase based machine translation (PBMT) (Chiang 2005) nor NMT (Bahdanau et al. 2014) are feasible for Bhojpuri, as such approaches require large parallel corpora. In their recent work, Sharma and Singh (2017) introduce a 'word transduction' approach to deal with the presence of unknown (or out of vocabulary) words for MT systems involving such resource-scarce languages. The concept of word transduction is somewhat similar to Hajič (2000), where the author suggests that the use of word-for-word translation for very closely related languages provides a good solution for MT of such language pairs.

Furthermore, for the task of language translation, it is necessary to take into account the fact that not all languages possess the same morphological features. For example, Finnish has more than 2000 possible inflected noun forms (Ekman and Järvelin 2007); Hindi and Bhojpuri have more than 40 inflectional forms (Singh and Sarma 2010); while English has a mere 7-8 (these numbers indicate the different possible valid combinations of morphological tags that nouns can possess). Therefore, a good MT system designed for such morphologically rich languages must be intricate enough to deal with their diverse inflectional morphology. In order to address this issue, we adapt character-level NMT systems to our task in order to ex-

${ }^{1}$ There is no consensus about the meaning of the word 'Hindi' and so different scholars have different views. For example, some other sub-languages like Rajasthani, Maithili and Magahi are also often included in the Hindi spectrum. However, the usual meaning of the word 'Hindi' in literature refers to standard Hindi, whose base is Khari Boli and which is an official language of India.

2 http://www.censusindia.gov.in/Census_Data_2001/Census_Data_ Online/Language/data_on_language.aspx 
ploit morphological information encoded in inter-character interactions and intra-word patterns. As observed by (Nakov and Tiedemann 2012): "character-level models combine the generality of characterby-character transliteration and lexical mappings of larger units that could possibly refer to morphemes, words or phrases, as well as to various combinations thereof" (Nakov and Tiedemann 2012). We also introduce a novel pre-trained character-level embedding (Bojanowski et al. 2017) for Devanagari alphabets derived from the 300 dimensional Hindi fastText embeddings. ${ }^{3}$

As regards the phonetic considerations of transduction, we make use of the fact that Hindi and Bhojpuri have a phonetic writing system, meaning there is an almost one-to-one mapping between phonemes (pronunciation) and graphemes (transcription). This is due to the fact that they both derive from common ancestor languages such as Prakrit and then Apabhramsha (Choudhury 2003). Hence, it suffices to work in either one of the spaces - orthographic or phonetic, and we choose the orthographic space since it does away with the need to convert the graphemes of text to and from phonemes.

Although Bhojpuri phonology is close to that of Hindi, it is not the same. There are notable differences between the two. While Hindi has a symmetrical ten vowel system, Bhojpuri has six vowel phonemes and ten vocoids. Similarly, Hindi has 37 consonants (including those inherited from earlier Indo-Aryan and those from loan words), whereas Bhojpuri has 31 consonant phonemes and 34 contoids. As is usual with any pair of languages, there are many phoneme sequences which are allowed in Hindi, but not found in Bhojpuri and vice-versa. This will be evident in examples given in the paper later. More details about the Bhojpuri phonology are available in the article by Trammell (1971).

\subsection{A note on Roman representations and English glosses of Hindi}

Throughout this paper, we have used the WX notation (Gupta et al. 2010) to represent (in a transliteration-like fashion) Hindi and Bhojpuri characters in English, for the benefit of readers who are not familiar with the Devanagari script. A ready reference table of the WX notation can be found in its Wikipedia page. ${ }^{4}$ Every non-English word

\footnotetext{
${ }^{3}$ https://github.com/facebookresearch/fastText/blob/master/ docs/pretrained-vectors.md

${ }^{4}$ https://en.wikipedia.org/wiki/WX_notation
} 
used in this paper is followed by its WX notation in square brackets ([]) and its italicized English gloss, in parenthesis (()).

\section{$1.2 \quad$ Transduction and translation}

Our usage of the word 'transduction' distinguishes it from translation, in that transduction is a task which is trained exclusively on cognates, and in that sense, the dataset it uses is a subset of the dataset that a translation system would use. Cognates are word pairs that not only have similar meaning but are also phonetically (and, in our case, orthographically) similar. The underlying observation that guides the usage of our proposed method of 'transduction' of OOV words as a possible substitute for their translation is as follows:

As stated earlier, Bhojpuri is a language closely related to Hindi. In the case of an OOV Hindi word (or any Hindi word for that matter), there is a good chance that the Bhojpuri translation of the word is a cognate of the Hindi word adapted to the phonological and orthographic space of Bhojpuri due to the presence of borrowed words, common origins, geographic proximity, socio-linguistic proximity, etc. A phonemic study of Hindi and Bundeli (Acharya 2015), mainly focusing on the prosodic features and the syllabic patterns of these two languages, (unsurprisingly) concluded that the borrowing of words from Hindi to Bundeli generally follows certain (phonological) rules. For instance if a word in Hindi begins with the character य [ya], it is replaced by character ज [ja] in its Bundeli equivalent as यजमान [yajamaan] (host) becomes जजमान [jajamaan], यमुना [yamunaa (name of a river)] becomes जमुना [jamunaa], etc. We observe that a similar process happens for Hindi-Bhojpuri. This category of word pairs is our main motivation behind the work described in this paper.

Our model is agnostic to what sort of words are considered to be OOV (based on their unigram probabilities, their parts-of-speech (POS), or whether named entities, etc.) because the above assumptions hold uniformly across the language pair. Section 2 specifies some of the metrics that have been used earlier to identify OOV words in related work.

Further, the above assumption (of transduction improving overall translation performance) has been demonstrated to be valid in the case of many closely related language pairs, in a number of previous works. For instance, Kondrak et al. (2003) extracted a parallel list of 
cognate word pairs and re-appended them to the parallel list of all word translations, thereby increasing the training weights of cognate words. Giving added importance to these cognate words, "resulted in a $10 \%$ reduction of the word alignment error rate, from $17.6 \%$ to $15.8 \%$, and a corresponding improvement in both precision and recall." (Kondrak et al. 2003). Mann and Yarowsky (2001) used cognates to expand translation lexicons, Simard et al. (1993) to align sentences in a parallel translation corpora, and Al-Onaizan et al. (1999) used cognate information to improve statistical machine translation.

Finally, transduction induces less sparsity in the model as compared to translation, because the hypothesis space is restricted to only functions that map words to their possible cognates. For closely related languages, the added reduction in sparsity also comes from the fact that there are consistent variations between how a source word transduces to its cognate target word. Hence, transduction is a task that performs better with a small training set than translation would when using a similarly complex model. This reduced sparsity enables transduction models to perform well on OOV words.

The ensuing section provides background about NMT systems and the manner in which we have adapted them to our task.

\section{Neural machine translation}

Neural machine translation (NMT) has delivered promising results in large-scale translation tasks such as Chinese-to-English (Tu et al. 2017) and English-to-French (Chen and Wu 2017). Initially, NMTs were used as a sub-component of the PBMT system such as for generating the conditional probabilities of phrase pairs (Cho et al. 2014), for generating (machine learning) features for the PBMT, or for re-ranking the $n$-best hypotheses produced by the system (Kalchbrenner and Blunsom 2013; Sutskever et al. 2014a). Such combined systems produced stateof-the-art results. One most appealing feature of NMTs is that they are largely memory efficient. Unlike PBMT systems, an NMT system does not require keeping track of phrase pairs or language models. Additionally, the work of Bentivogli et al. (2016) pointed out that NMTs offer a range of other superior attributes including:

- generation of outputs that require considerably lower post-edit efforts 
- better translation quality in terms of BLEU score, Translation Edit Rate, and good performance on longer sentences

- fewer and/or less-severe errors in terms of morphology and word order

Most NMT systems today make use of the encoder-decoder based approach (e.g. Forcada and Ñeco 1997; Cho et al. 2014; Kalchbrenner and Blunsom 2013; Sutskever et al. 2014a), which consists of two recurrent neural networks (or their variants). The first encodes a variable-length source token $x$ into a fixed length vector and the second decodes the vector into a variable-length target token $y$. NMT approaches were initially designed to work at the word-level and translate sentences. However, noting the encouraging results of adapting NMTs to character-level translation by Vilar et al. (2007), we adapt NMTs to our character-level transduction. Figure 1 shows the architecture of such an encoder-decoder based NMT system performing character-level transduction. The model is trained over a parallel corpus to learn the maximum conditional probability of $y$ given $x$, i.e., $\arg \max _{y} p(y \mid x)$. Once trained, the model can then be used to generate the corresponding transduction for a given source word.

However, the performance of NMT degrades largely in the case of longer length sequences (words, in our case) due to the vanishing

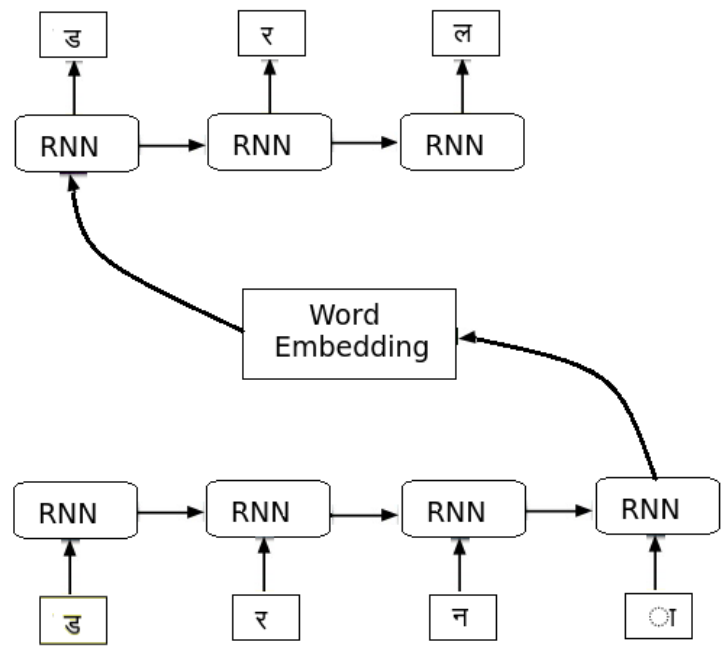

Figure 1:

Encoder-decoder network architecture transducing the Hindi word डरना [darnA] (to be scared) to Bhojpuri डरल [darala] 
gradient problem (Bengio et al. 1994) arising during the training of the underlying RNN. So far, the use of an attention mechanism, as stated by Bahdanau et al. (2014), Luong et al. (2015a), Vinyals et al. (2015) and Yang et al. (2016) has been the most plausible solution to the aforementioned problem for RNNs and its variants. The concept of 'attention' takes into account the fact that in the task of translation, different tokens in a sequence are differentially informative, with the information carried by them being highly context dependent. Thus, for predicting each corresponding token, the model looks at the current context of the source token that is relevant to predicting the target token.

\section{4}

Summary

We adapt NMT models to perform 'transduction' of a Hindi word to a Bhojpuri word. These word-transduction models work with characters as the fundamental units. They are trained on Hindi-Bhojpuri cognate pairs. This task is important because it helps to solve the OOV problem in larger downstream tasks, the most prominent example of which is machine translation for low resource languages. To improve machine translation of Hindi to Bhojpuri, we first identify OOV words in Hindi texts and then use our model to transduce them to their Bhojpuri counterparts. All other words are translated, and not transduced. Using such separate treatment of OOV words, we obtain improvements in BLEU score with respect to the originally translated texts. The section on Related Work (Section 2) elaborates previous approaches to transduction and the OOV problem.

A number of methods have been proposed to handle the OOV problems in machine translation of unknown or rare words. Luong et al. (2015a) simply use a shortlist of 30,000 most frequent words and map all other less frequent words to an UNK (unknown) token. Sutskever et al. (2014b) use a vocabulary of 80,000 words and achieve better performance. However, any UNK-based approach is problematic because in larger sentences, UNK tokens heavily degrade performance (Cho et al. 2014; Jean et al. 2015) make model specific improvements, using a smaller batch for normalization and including only frequent 
words in the denominator of this normalization. They fall back to other translation and alignment models to replace UNK tokens.

Other approaches to handle OOV words include using a backoff dictionary look-up (Jean et al. 2015; Luong et al. 2015b) but as observed by Sennrich et al. (2016), these techniques make impractical assumptions. One such assumption is a one-to-one source - target word correspondence, which is unwarranted. Further, some of these methods assume the existence of a parallel corpus of source-target word pairs, which is not always available in the case of low resource languages. Sennrich et al. (2016), in turn, use a Byte-Pair Encoding for transduction, which is very similar to character-level encoding of sequences as strings of characters.

We also borrow ideas from previous approaches that have used cognates. Simard et al. (1993) use cognates to align sentences in a parallel corpus and report $97.6 \%$ accuracy on the alignments obtained, when compared to reference alignments. Mann and Yarowsky (2001) use cognates extracted based on edit distances for inducing translation lexicons based on transduction models. Scannell (2006) presents a detailed study on translation of a closely related language pair, IrishScottish Gaelic. They learn transfer rules based on alignment of cognate pairs, and use these rules to generate transductions on new words. They use a fine-grained cognate extraction method, by first editing Scottish words to 'seem like' Gaelic words, and then using edit string similarity on the new word pairs and choosing only close words with the additional constraint that both words in the pair should share a common English translation. However since we use linguistic experts to extract cognates from our dataset, we do not need to encode string similarity measures explicitly to extract cognates.

We borrow insights from character-level machine transliteration and translation models that have been proposed in the past, as transduction can be viewed as a variation of transliteration (which is, in turn, viewed as character-level translation in many works), albeit working within the same script. Alternatively, it can also be thought of simply as a translation of 'true friend' cognates.

Vilar et al. (2007) work on transliteration at the character level (and translation at the word level) to build a combined system that shows increasing gains over just the word-level system, as the corpus size grows smaller. This is because the character-level transliter- 
ation takes into account the added morphological information such as base forms and affixes. Tiedemann (2012) experimented with different types of alignment methods and learning models, and showed that in each type of method, there exists at least one character-level model that performs better than word-level models (in the case of closely related language pairs). Denoual and Lepage (2006) also show merits of using characters as appropriate translations, and highlight issues with making assumptions about words being natural units for the task. Finch and Sumita (2009) view transliteration as a characterlevel machine translation, and use Phrase-Based SMT for bidirectionally encoding source sequences. They observe the lack of necessity to model phonetics of source or target language, due to the use of direct transformations. One point of difference between some of the related work on cognates and ours is that we do not perform contextsensitive transduction simply due to lack of annotated data that is context-sensitive.

We run experiments on four different benchmark encoder-decoder networks, namely:

- a simple sequence-to-sequence model (Cho et al. 2014) - abbreviated as 'seq2seq'.

- the alignment method (Bahdanau et al. 2014) incorporated with the seq2seq model - abbreviated as 'AM'.

- the Hierarchical Attention Network (Yang et al. 2016) incorporated with the seq2seq model - abbreviated as 'HAN'.

- the Transformer Network (Vaswani et al. 2017) solely based on attention - abbreviated as 'TN'.

In the basic seq2seq RNN encoder-decoder model (Cho et al. 2014) we incorporate a 'peek' at the context vector at every time step. However, the model performed poorly on this translation task, with the validation accuracy plateauing at a low value early on in the training process. Section 3.1, Section 3.2 and Section 3.3 describe the models - AM, HAN and TN, respectively - in detail. 


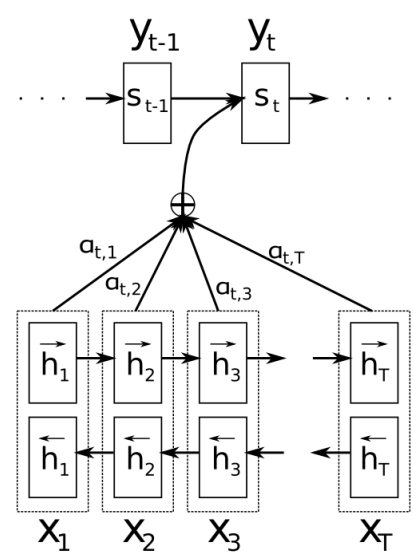

Figure 2:

Schematic of the alignment model adapted from Bahdanau et al. (2014)

As shown in Figure 2, the alignment model (AM) proposed by Bahdanau et al. (2014) facilitates searching through the source sequence during the decoding phase using a unique context vector for each token. Specifically, given a translation $y_{i}$ and the source sequence $\mathbf{x}$, the decoder decomposes the conditional probability over all the previously predicted tokens $\left(y_{1}, \ldots, y_{i-1}\right)$ as:

$$
p\left(y_{i} \mid y_{1}, \ldots, y_{i-1}, \mathbf{x}\right)=g\left(y_{i-1}, s_{i}, c_{i}\right),
$$

where $s_{i}$ is the hidden state of the decoder model computed for time $i, c_{i}$ is the distinct context vector for each target token $y_{i}$ and $g$ is a non-linear function that outputs the probability of $y_{i}$ being the correct translation at time $i$.

In addition to the use of a unique context vector for each decoding time step, all hidden states computed so far contribute to the context vector $c_{i}$ with weight $\alpha$.

$$
c_{i}=\sum_{j=1}^{T_{x}} \alpha_{i j} h_{j}
$$

$\alpha$ thus serves as a normalized importance weight, measuring the degree of importance of the context tokens around position $j$ in predicting the translation of the current source token at the output position $i$ (Bahdanau et al. 2014). Figure 2 shows the architecture of the encoder-decoder network incorporating the alignment-based attention decoder. 
Figure 3:

Encoder-decoder network architecture with HAN: $n$ and $m$ denote the number of characters in input and output words respectively

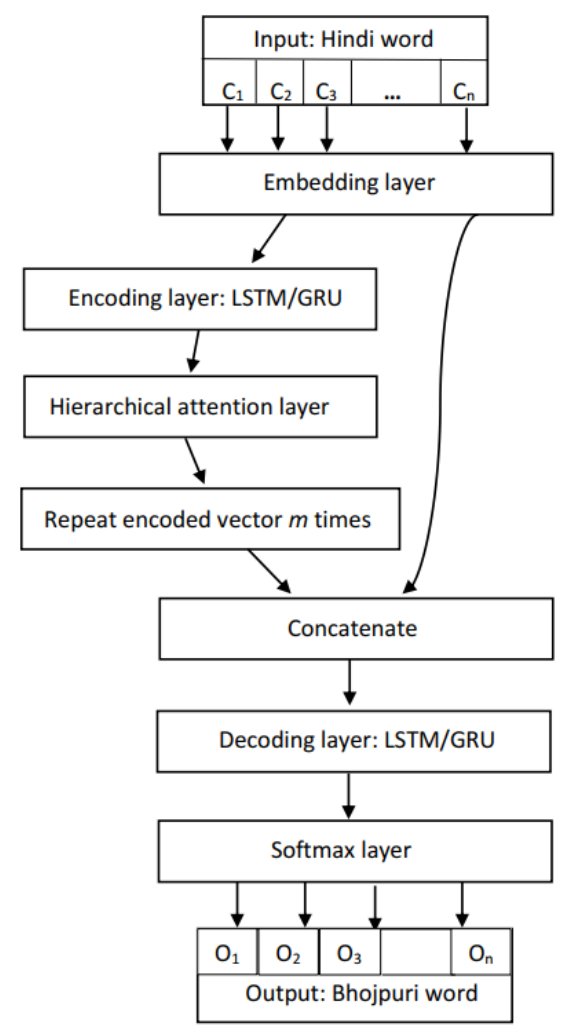

Proposed by Yang et al. (2016), Hierarchical Attention Networks (HAN) exploit the hierarchical nature of documents (i.e., characters form words, words form sentences and sentences form a document) and are comprised of two levels of attention mechanisms (Bahdanau et al. 2014; Lai et al. 2015) - the first at the word level while the other at the sentence level. In our case, the former attention can be thought of as being effective at the character level, while the latter at the word level, thus allowing the model (Figures 3 and 4) to discover the amount of attention required to be paid to the individual characters and words to form a character-level transduction.

Self-attentional transformer network (TN)

The Transformer Network (Vaswani et al. 2017) consists of an encoder made up of a stack of six identical layers. Each layer further con- 


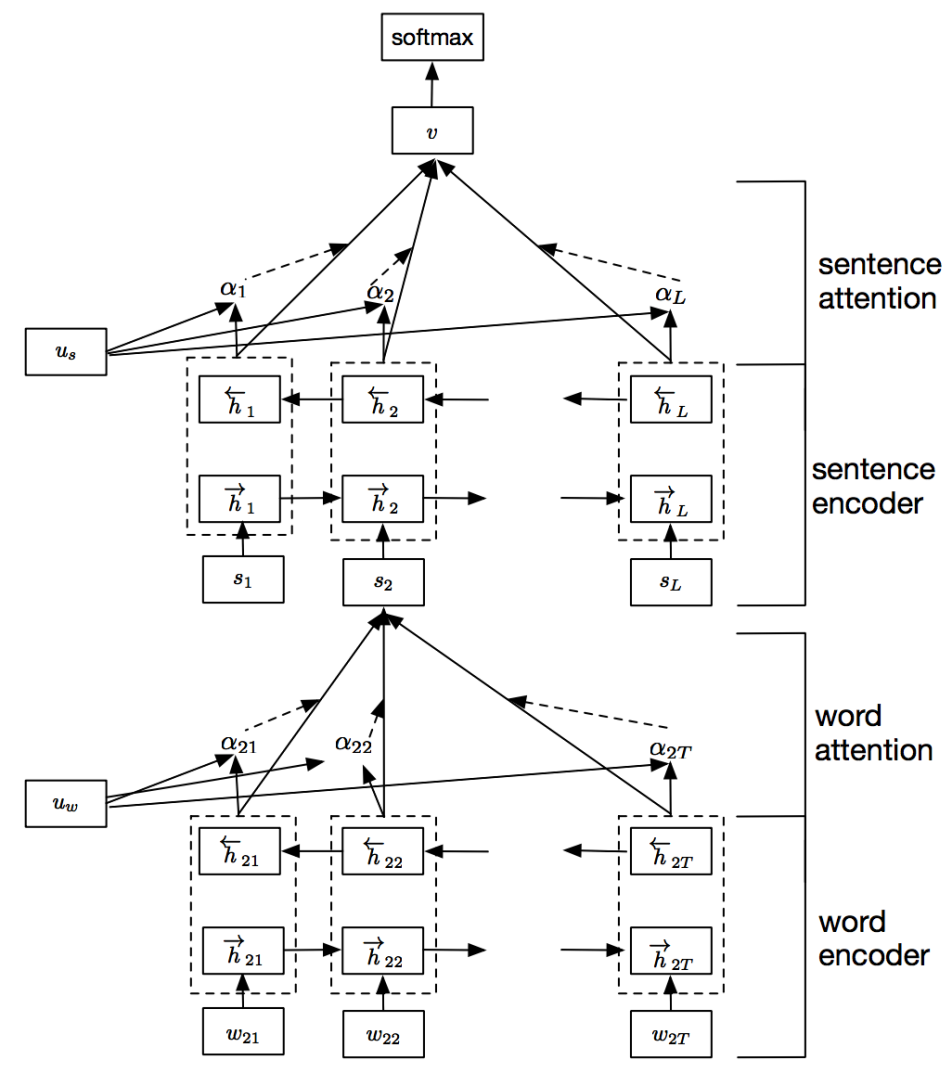

Figure 4:

The HAN framework adapted from Yang et al. (2016)

sists of two sub-layers: a multi-head self-attention and a simple positionwise fully connected feed-forward network (FFN). The decoder too has a similar architecture except for an additional sub-layer performing multi-head attention over the output of the encoder stack. Both the encoder and the decoder unit employ a residual connection (He et al. 2016) in between their respective sub-layers, followed by layer normalization (Ba et al. 2016).

As shown in Figure 5, the positional embeddings serve to make the representation at time step $i$ independent from the other time steps. The multi-head attention layer serves to replace recurrent dependencies by repeatedly applying self-attention over the same inputs using separate parameters (attention heads) followed by combining the results. This combination acts as an alternative to applying a single pass of attention with more parameters so that the model can easily learn 
Figure 5:

The transformer architecture as described in Vaswani et al. (2017) and adapted from Li et al.

(2018)

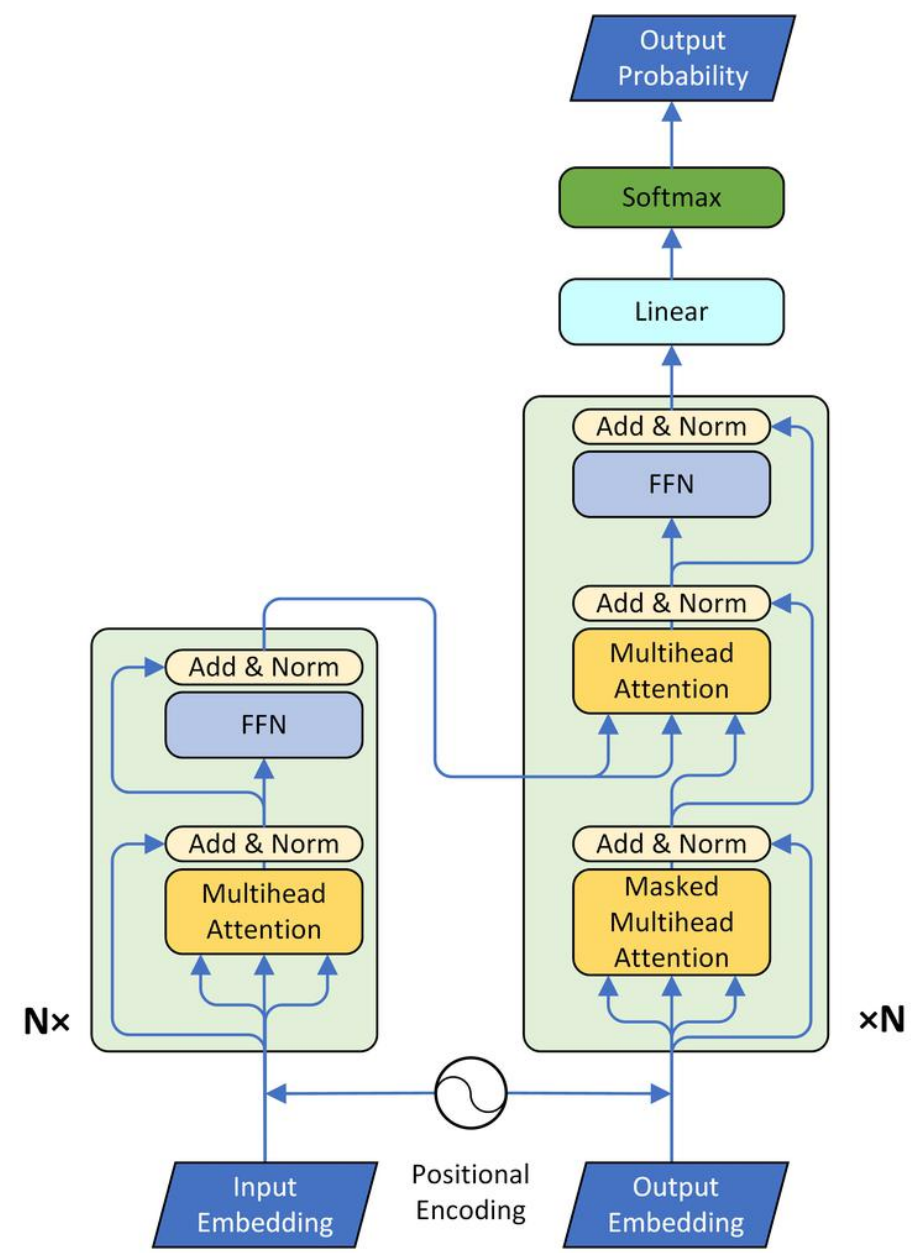

to attend to different types of relevant information in parallel with each head. In other words, the decoder can now use multiple encoderattention mechanisms in each of its layers resulting in a significantly faster training than architectures based on recurrent or convolutional layers. Inspired by the success of the Transformer in sequence generation tasks (such as achieving state-of-the-art results on both WMT2014 English-German and WMT2014 English-French translation tasks ${ }^{5}$ ), we use the Transformer Network in our task.

\footnotetext{
${ }^{5}$ http://www. statmt.org/wmt14/
} 
For the AM and HAN models, we consider various parameters while training, such as LSTM/GRU as encoding/decoding units, sequence chunking and batch sizes, optimization methods, regularization, and we report that there is a huge variance in the transduction performance depending on the combinations of the parameters used (for details of these experiments, the interested reader may refer to the Appendix section in the pre-print of this work ${ }^{6}$ ). As regards the TN model, due to computational and implementation limitations, we could not perform such an extensive hyperparameter search. Nevertheless, we take care of the basic parameter settings (as suggested in Popel and Bojar 2018) by limiting our model to a single-GPU base, setting batch size to 512, maximum sequence length to the length of the longest token in the parallel corpus followed by a final averaging of the last 6 training checkpoints while leaving the learning rate and the number of warm up steps at their default values. Finally, the extracted character embeddings are used to train the AM and HAN models while the TN model is left void of these mainly because of its inherent dependency on segmenting the training tokens into semantically useful sub-tokens which are hard to be reproduced in varying experiments (Popel and Bojar 2018), and thus cannot be easily assigned such embeddings. We compare the results of the TN model with the best results (among various hyperparameter settings) of the AM and HAN models.

The implementation of the Alignment model (AM) and the Hierarchical attention network (HAN) is based on Keras-2.0.6 (Chollet et al. 2015); that of the Transformer Network (TN) is based on tensorflow-1.4.1 (Abadi et al. 2015) and tensor2tensor-1.4.3. ${ }^{7}$ Experiments were run on x86_64 GNU/Linux with 8G memory, using one NVIDIA GeForce 840M with CUDA v8.0.61, and Python 3.5.2+.

4.1

\section{Dataset}

In order to be able to compare our results with the state of the art (SOTA) - described in Section 4.3 - we use the same dataset as Sharma and Singh (2017), which was the state-of-the-art method at the time of writing. This dataset consists of 4220 Hindi-Bhojpuri word

\footnotetext{
${ }^{6}$ https://arxiv.org/ftp/arxiv/papers/1811/1811.08816.pdf

7 https://github.com/tensorflow/tensor2tensor
} 
cognate pairs chosen from a pre-compiled lexicon of Hindi-Bhojpuri word translations. This dataset was compiled by three linguistic experts (who are native speakers of both Bhojpuri and Hindi) who came to consensus on the annotations. Cognate pairs were identified from this set using domain expertise by linguistic experts. This method of cognate extraction is in contrast to possibly sub-optimal rule-based and similarity-based approximations, such as those used by Mann and Yarowsky (2001). In summary, the training data has Hindi-Bhojpuri word pairs, each of which comprises a Hindi and a Bhojpuri word that have the same meaning, as well as similar pronunciations. We split the dataset into 3:1 ratio for training and testing our models. A validation split of 0.1 was further made on the train set comprising of 3165 word pairs. This test is held-out and is used for reporting only the final results. All hyperparameter tuning is done on the validation set. The validation set is not fixed and the training is crossvalidated, with a new random validation-set being used at each iteration of tuning. Table 1 consolidates these statistics. We perform a random shuffle of the train and validation set prior to each training epoch.

Table 1:

Transduction corpus statistics

\begin{tabular}{r|ccc}
\hline & Training set & Validation set & Test set \\
\hline Total number of words & 2849 & 316 & 1055 \\
\% of words of full dataset & $67.5 \%$ & $7.5 \%$ & $25 \%$ \\
\hline
\end{tabular}

4.1.1 Semantic ambiguity when selecting cognates

It is worthwhile to mention that not all Hindi words in the training dataset have just one possible corresponding Bhojpuri cognate. Around $3.85 \%$ of Hindi words in the Hindi-Bhojpuri cognate pairs have more than one corresponding Bhojpuri cognate. The same applies the other way too, i.e., not all Bhojpuri words have just one possible corresponding Hindi cognate. Around $3.37 \%$ of Bhojpuri words in the Hindi-Bhojpuri cognate pairs have more than one corresponding Hindi cognate. When either a Hindi word or a Bhojpuri has multiple corresponding cognates, only two possible cases arise, as described next. 
1. The multiple possible cognates have the same semantic sense and are different in only surface forms (spellings). For instance, the Bhojpuri word हलुवा [haluVA] (a sweet dish) has two Hindi cognates - हल्वा [halVA] and हलवा [halaV] - both meaning the same, but differing in surface forms due to the presence of the diacritic in one and absence in the other. The diacritic is a character explained in Section 6.1.1.

2. They are different inflected forms of the same root word. For instance, the Bhojpuri word राखल [rAKala] (kept, or to keep) has two Hindi cognates - रखा [raKA] (kept) and रखना [raKanA] (to keep) - both of which are different inflected forms of the root word रख [raKa] (keep).

Since multi-cognate words together form only a small percentage of the dataset, they are not accorded any special treatment, and the model learns from the multiple forms.

We plan to work more on the deeper change in ambiguity from source to target word in the case of 'true friend' cognates, particularly for the Hindi-Bhojpuri language pair.

We report the accuracy of each experiment using the BLEU score and Levenshtein distance-based string similarity (SS) measure, as in Equation $3 .^{8}$ After obtaining the optimum hyperparameter set for AM and HAN, we compare the word accuracy (WA, Equation 4) report defined by the percentage of correctly translated words for all the models including the SOTA. SS, WA and BLEU score formulae for two arbitrary strings ' $s 1$ ' and ' $s 2$ ' are given below. The averaged score across the validation/test set are reported in the tables in ensuing sections. We employ the character $n$-grams version of BLEU score (as used in Denoual and Lepage 2005) as our work is at the word-level, instead of document-level. Using this metric also alleviates the comparison with other state-of-art transduction methods since other popular metrics such as CHRF and TER correlate well with the character $n$-gram version of BLEU score (as observed by Popović 2015).

\footnotetext{
${ }^{8}$ https://pypi.python.org/pypi/python-Levenshtein/๑.12.๑
} 


$$
S S(s 1, s 2)=\left(1-\frac{\text { Levenshtein Edit Distance }(s 1, s 2)}{\operatorname{len}(s 1)+\operatorname{len}(s 2)}\right) * 100
$$

$$
W A(s 1, s 2)=\{1 \text { if } s 1==s 2 ; 0 \text { otherwise }\}
$$

4.3

State of the art

We consider the results of Sharma and Singh (2017) to be state of the art, and to the best of our knowledge, the only relevant one on Hindi-Bhojpuri transduction or even any form of OOV word handling technique for this language pair. While their work builds upon traditional PBMT approaches, they first convert lexical word representations into phoneme strings followed by the alignment of phonemes in these strings. The word is then segmented into phoneme chunks which thus facilitates the extraction of weighted rewrite rules for these chunks.

Since the work of Sharma and Singh (2017) extensively compares and contrasts their own work to the related work in transduction, we refrain from such an elaborate comparison, and suggest their work to the reader for more comparisons to other techniques. We show improvements in performance over their results.

Our adaptations of each of the models (i.e., seq2seq, AM, HAN and TN) use Bidirectional LSTMs (BLSTM) as encoder-decoder units unless specified otherwise. A detailed analysis of hyperparameter tuning and training aspect for each model can be found in the Appendix section in the pre-print of this work. ${ }^{9}$ The analyses document the experiments motivating our decisions on using LSTM vs GRU, sizes of encoding and decoding layers, number of layers, batching, optimization methods, regularization methods and pre-training embeddings for each of the three models. We hope that these results may be useful for future work in morphology-related tasks.

4.5

Character embeddings

Since the atomic token in our models is the character (and not the word), we explore two pre-training strategies - 'LM-LSTM-Embed' and

\footnotetext{
${ }^{9}$ https://arxiv.org/ftp/arxiv/papers/1811/1811.08816.pdf
} 
'fT-Avg-Embed' - to represent characters as dense vector embeddings. These embeddings are used only as pre-trainings, and the transduction models are allowed to update these weights during training, i.e., they are not frozen. The followings sections describe methods used to create these embeddings.

\subsubsection{Creating embeddings using LSTM}

Taking cues from Sundermeyer et al. (2012), we use a simple onelayered Bi-LSTM of hidden dimension 75 and a dropout of 0.5 to train a character-level language model for Hindi. Working at the characterlevel, we formulate the problem as a character prediction task - given a sequence of 29 characters, predict the next one, i.e., the $30^{\text {th }}$ character. We found 30 to be the optimum window size after varying window sizes from 5 to 40, keeping in mind memory requirements as well as perplexity. We randomly sample 3000 Hindi Wikipedia articles as a train set (with a validation split of 0.2 ) and another random 600 articles as a held-out test set. With a train set size of over 19M characters, a vocabulary of 397 distinct characters (including special characters and code-mixed characters ${ }^{10}$ ), and having trained for 31 epochs monitored upon validation loss convergence with a patience of 7 epochs, the model resulted in a perplexity score of 5.18 over the test set. The weights of the character embedding layer were then extracted to be used as pre-trained yet trainable character embeddings for our transduction models. We refer to this method of pre-training as 'LM-LSTMEmbed'. The embedding weights are allowed to be trained along with the hyperparameters of the models as freezing these lead to a decrease in performance - a plausible reason being the orthographic and grammatical distinction of Hindi (on which the character embeddings are based) from Bhojpuri (the target language whose orthography and grammar must be reflected by modifying the pre-trained embedding weights).

\subsubsection{Creating embeddings by averaging fastText embeddings}

While using the LM-LSTM-Embed embeddings gives the best performance, we also introduce a novel pre-trained character-level embeddings set for the Devanagari script; these are derived from the 300-D

\footnotetext{
${ }^{10}$ Characters borrowed from other languages.
} 
Hindi fastText embeddings (Bojanowski et al. 2017). We choose the fastText embeddings over other benchmark embeddings because of their inherent sub-word information preserving property that arises from representing each word as a bag of character $n$-grams ${ }^{11}$ and not as an atomic token itself. As has been observed by Bojanowski et al. (2017), such subword-level representation of words is highly useful in capturing morphological structure of words. We start with the existing word embeddings for Hindi. ${ }^{12}$ These fastText embeddings have been constructed for each word by averaging the embeddings of the character $n$-grams that make up the word. We derive the pre-trained embedding of a character by averaging over all word vectors of words in which the character occurs, weighted by the number of times it occurs in each word. We refer to this method of pre-training as 'fT-Avg-Embed'. We outline the reason to support this method.

For character $n$-grams, in the case when $n=1$ (and loosely extending for $n>1$ ), each existing word embedding would have been the average of all its characters' embeddings (or when $n>1$, groups of characters' embeddings). An approximation of 're-obtaining' the character embeddings from the word embeddings would then be to average over all word vectors of words in which the character occurs. This linear transformation would preserve the 'component' of the embedding of the particular character, as the components of all the other characters would cancel each other out. This justification also takes into account the fact that the components of all characters that are not 'close' (in the sense of co-occuring in the same context, i.e., word) to the particular character would cancel out each other since their vectors can be thought of as being spatially randomly distributed with respect to the vector of the character in question. This also implies that the resulting character embedding will have contributions from the components of characters that are 'close' to it. We propose this method of computing character embeddings as follows.

\footnotetext{
${ }^{11}$ Consequently, we observe that using the fastText derived embeddings provides highly consistent results in comparison to using GloVe (Pennington et al. 2014) or word2vec (Mikolov et al. 2013) character adaptations over monolingual Hindi corpora.

12 https://github.com/facebookresearch/fastText/blob/master/ docs/pretrained-vectors.md
} 
NMT word transduction mechanisms for LRL

\begin{tabular}{r|ccc|ccc}
\hline \multirow{2}{*}{ Initialization methods } & \multicolumn{3}{|c|}{ AM } & \multicolumn{3}{c}{ HAN } \\
& BLEU & SS & ep & BLEU & SS & ep \\
\hline ft-Avg-Embed & 87.32 & 85.56 & 59 & 83.14 & 80.89 & 21 \\
LM-LSTM-Embed & $\mathbf{8 9 . 7 1}$ & $\mathbf{8 8 . 0 3}$ & 22 & $\mathbf{8 5 . 9 4}$ & $\mathbf{8 4 . 0 5}$ & 17 \\
Random & 73.51 & 71.68 & 16 & 64.11 & 61.33 & 12 \\
Zero & 68.44 & 63.10 & 14 & 60.83 & 59.20 & 13 \\
\hline
\end{tabular}

Table 2:

Effect of pre-trained character embeddings: $e p$ indicates the number of epochs until convergence

(a) It allows us to circumvent the need for extensive lexical and computational resources required for training character embeddings on a large Hindi monolingual corpus from scratch (while making small compromises on overall downstream accuracy).

(b) It uses pre-existing vectors that have been successfully tested upon a range of tasks (Chaudhary et al. 2018), lending the method of deriving character embeddings (from word embeddings) in this manner to any pre-trained word vectors that have been themselves composed from sub-word representations.

(c) It provides scope to study the notion of character embeddings, vis-a-vis word embeddings, since the semantic notion of a word and its embedding is well understood but the notion of a dense representation of a character is not fully understood yet.

It is important to note that pre-training using the LM-LSTMEmbed method results in the best transduction performance, and also that using the fT-Avg-Embed pre-trainings does significantly better than random initializations, while incurring lesser computation costs than LM-LSTM-Embed. These differences are shown in Table 2 for Hindi-Bhojpuri and in Table 3 for Hindi-Bangla (additional experiments on this language pair will be described in Section 5.3). In both these tables, we observe that the performance improvements across the metrics alongside the increase in training epochs before convergence of the models remain consistent, i.e., LM-LSTM-Embed is a better pre-training strategy than ft-Avg-Embed.

RESULTS

5.1 Comparing our models to the state-of-the-art model

Table 4 compares the performances of the best AM and HAN models (after the hyperparameter search) with those of the standard encoder- 
Saurav Jha, Akhilesh Sudhakar, Anil Kumar Singh

\begin{tabular}{|c|c|c|c|c|c|c|c|c|}
\hline \multirow{6}{*}{$\begin{array}{r}\text { Table 3: } \\
\text { Effect of pre-trained } \\
\text { character embeddings for } \\
\text { Hindi-Bangla cognate pairs }\end{array}$} & \multirow{2}{*}{\multicolumn{2}{|c|}{ Initialization methods }} & \multicolumn{3}{|c|}{ AM } & \multicolumn{3}{|c|}{ HAN } \\
\hline & & & BLEU & SS & ep & BLEU & SS & ep \\
\hline & \multicolumn{2}{|c|}{ ft-Avg-Embed } & 78.49 & 77.10 & 56 & 73.65 & 70.22 & 26 \\
\hline & \multicolumn{2}{|c|}{ LM-LSTM-Embed } & 81.03 & 79.72 & 33 & 75.28 & 73.15 & 24 \\
\hline & \multicolumn{2}{|c|}{ Random } & 72.66 & 72.25 & 19 & 60.07 & 58.46 & 10 \\
\hline & \multicolumn{2}{|c|}{ Zero } & 70.39 & 69.88 & 19 & 59.21 & 55.33 & 12 \\
\hline \multirow{4}{*}{$\begin{array}{r}\text { Table 4: } \\
\text { Comparison of evaluation } \\
\text { metrics among } \\
\text { encoder-decoder models }\end{array}$} & Metric (\%) & \multicolumn{2}{|c|}{ seq2seq } & $\mathrm{AM}$ & HAN & $\mathrm{TN}$ & \multicolumn{2}{|c|}{ SOTA } \\
\hline & BLEU & \multicolumn{2}{|c|}{52.89} & 89.71 & 85.94 & 90.89 & \multicolumn{2}{|c|}{79.82} \\
\hline & SS & \multicolumn{2}{|c|}{57.22} & 88.03 & 84.05 & 90.23 & \multicolumn{2}{|c|}{-} \\
\hline & WA & \multicolumn{2}{|c|}{16.32} & 67.22 & 59.77 & 75.7 & \multicolumn{2}{|c|}{64.41} \\
\hline
\end{tabular}

decoder model, the TN model and the current state-of-the-art (SOTA) model (Sharma and Singh 2017). The BLEU scores of AM, HAN and TN are higher than that of the phoneme-chunk based model used by Sharma and Singh (2017). While TN outperforms the SOTA model in terms of word accuracy (WA), AM and HAN lag behind. The simple seq2seq model performs the worst among all our models, and fails to match up to SOTA.

TN performs the best due to two main reasons:

1. Residual connections that connect the input character embeddings to the final decoder of the output word: The transduction of a Hindi word can be thought of as making character level edits upon the Hindi word, in the same orthographic space. The residual connections, hence, help in learning these edits by conditioning the final decoder not only on the attention-based representation of the input word but also directly the input word itself.

2. The multi-head attention mechanism of TN helps to model the dependencies of characters in the input and output words, regardless of their distances from each other. This would otherwise have to be learnt from a restricted fixed-sized representation, which is usually an LSTM.

The simple seq2seq model performs the poorest because, perhaps, the generic architecture is not adapted to the task in any manner, based on knowledge about the linguistic properties between cognatepairs. This simple architecture fails to capture long-range dependencies for 1) longer words, and 2) words in which the dependencies be- 
tween orthographic segments in the source and target word are not very obviously aligned.

The performance improvements of HAN and AM could be attributed to their attention mechanisms that facilitate better capturing of the intricacies of phonetic and orthographic dependencies of cognates. It is interesting to see that HAN performs worse than the $\mathrm{AM}$, and this might be because of the over-parametrization of HAN. HAN is over-parameterized since it was originally proposed for building a hierarchy over documents where a layer of attention is effected across words in sentences of the document, and another layer is effected across characters in words of sentences. In our case we only deal with individual words, and not documents. This, combined with the fact that we have a small training set, perhaps causes HAN to overfit to the training data and perform poorly on the test set. We observed that the HAN transductions commonly had erroneous repeated characters at the ends of words; we thus employ a post-processing step to remove all but the first recurring character appended to these words. This will be referred to in later sections as the 'post-processing' step.

An elaborate account of errors made on different word-pair types by each of these models is presented in Section 6 . These differences in errors occur due to the differences in the models as expounded above.

Gains over the state of the art

Table 4 depicts the performance of the aforementioned models using both the pre-trained character embeddings obtained using averaging (AVG) and that built from the language model (LM). It is evident that TN performs best across all accuracy metrics. This is our best model and using this model achieves gains over SOTA of 11.07 BLEU score points (a percentage gain of 13.9\%) and a Word Accuracy gain of $11.3 \%$ (a percentage gain of $17.5 \%$ ). The SOTA paper did not provide information of SS scores, and hence we have not made this comparison.

\subsection{Additional experiments on Hindi - Bangla cognate pairs}

We extend our experiments to transducing from Hindi to Bangla by training on a corpus of Hindi-Bangla cognate pairs comprised of 3220 word pairs. We carried out the same 3:1 split upon this corpus to hold out the test set while making a further split of 0.1 upon the train set 
Saurav Jha, Akhilesh Sudhakar, Anil Kumar Singh

Table 5:

Comparison of evaluation metrics between seq2seq, AM, HAN, and TN for Hindi-Bangla cognate pairs

\begin{tabular}{r|cccc}
\hline Metric (\%) & seq2seq & AM & HAN & TN \\
\hline BLEU & 41.88 & 78.49 & 73.65 & $\mathbf{8 3 . 7 6}$ \\
SS & 55.12 & 77.10 & 70.22 & $\mathbf{8 2 . 5 9}$ \\
WA & 9.87 & 59.27 & 47.19 & $\mathbf{7 1 . 1 1}$ \\
\hline
\end{tabular}

(2415 word pairs) to obtain a validation set. The results of the Hindi - Bangla experiments are presented in Table 5.

The decline in the scores of all four models across the metrics in Table 5 (compared to Table 4) could be boiled down to two major reasons:

1. Word formation methods: Bangla has its roots in the Prakrit or middle Indo-Aryan language, which in turn descended from the old Indo-Aryan language, of which Sanskrit is a standardized form. Hindi also shares roots with Sanskrit. Bangla is therefore neither a dialect nor an immediate sibling of Hindi. This is unlike Bhojpuri. This also means that Bangla, which has its own Brahmi-derived script (namely, the Bangla script) has possible word formation rules that are quite different from Hindi. One such instance is the Bangla consonant clustering mechanism. For example, the name Vishnu, written as विष्णु [viRNu] in Hindi, has the consonant cluster $s a+n a[\mathrm{RN}]$. While the Hindi consonant cluster (ष + ण) can easily be decomposed into its constituent letters, the Bangla cluster can form a new character in itself.

2. Smaller size of the training corpora: The training corpora for HindiBangla cognate pairs comprises 2415 word pairs, i.e., 1000 instances less than that of Hindi-Bhojpuri train set. The aforementioned grammatical restrictions shared by Hindi and Bangla make it very demanding to discover more such cognate pairs between the two languages, thus constraining the training corpora for our experiments.

6

\section{ERROR ANALYSIS}

We analyse the outputs of each model to study a pattern in the most common errors made by each of them. We identify six types of orthographic and lexical errors, and four types of errors related to overall 
translation quality for a word. While orthographic errors are motivated with respect to the types of graphemes generated by character patterns, quality-related errors focus on overall aspects of the transduction being close to the correct translation. Further, we have been able to make some preliminary correlations between the model architectures (AM, HAN, TN) and the errors they make. The main weakness of the AM model is that since it is predominantly bidirectional LSTMbased and only weakly attention-based as compared to HAN and TN, it tends to bias character predictions towards either the early characters or the later characters in the input sequence, sometimes giving poor results towards the mid-sections. Since it processes input in a sequential manner, it also tends to lose out some orthographic information in the process. The TN model's weakness lies in the fact that it is infamously bad at performing copy mechanisms (Dehghani et al. 2018), and hence it fails in places where characters/character-groups have to be preserved in the transduction. The HAN model's trade-off between the LSTM's influence and the attention weights' influence lies between the AM and the TN, and the behaviour it shows with respect to errors it leads to, reflects this is in certain ways. However, these are only approximate inferences that we make retrospectively, with the actual behaviour varying on a case-by-case basis.

6.1

6.1.1
Orthographic and lexical errors

Halant

Halants refer to diacritics used to signify the lack of an inherent vowel in written Devanagari scripts. In Devanagari, the halant is represented by a diacritic below the consonant it applies on (e.g., द), while it is represented by not using any vowel after the consonant it applies on in the WX notation (e.g., द् is represented as simply ' $x$ '). In most of the cases, halants are preserved during translation of Hindi to Bhojpuri words, except a few (e.g. प्रथा [praWA] (tradition) becomes परथा [paraWA]). We study the capability of each model to handle the translation of halants (see Table 6). While HAN and TN perform reasonably well at translating halants, AM tends to replace the character possessing halant with some ligature combined with a neighbouring character, e.g. त्क [wka] in पत्कारनन [pawkAranana] and त्त [wwa] in पवित्ता [paviwwA]. The possible reason for this could be the better attention capabilities of the HAN and the TN, which helps these models force the 
Table 6: Handling halant

\begin{tabular}{|c|c|c|c|c|}
\hline Hindi & Bhojpuri (correct) & $\mathrm{AM}$ & HAN & $\mathrm{TN}$ \\
\hline प्रथा & परथा & पररा & परथा & परथा \\
\hline [praWA] & [paraWA] & [pararA] & [paraWA] & [paraWA] \\
\hline (tradition) & & & & \\
\hline पवित्रता & पवित्रता & पवित्ता & पवित्रत & पवित्रता \\
\hline [paviwrawA] & [paviwrawA] & [paviwwA] & [paviwraw] & [paviwrawA] \\
\hline (purity) & & & & \\
\hline पत्रकारों & पत्रकारन & पत्कारनन & पत्रा & पत्रकारन \\
\hline [pawrakAroM] & [pawrakArana] & [pawkAranana] & [pawrA] & [pawrakArana] \\
\hline (journalists) & & & & \\
\hline
\end{tabular}

halant to be appended in the right place, thus ensuring that the immediate neighbouring character of the halant in Hindi is joined with the previous character in the Bhojpuri transduction. The AM, having only limited attention influence in comparison, skips the immediate neighbouring character and combines the halant with a later character as the LSTM layer in the AM has seen the later character more recently.

\subsubsection{Handling vowels}

Vowels play an important role in the translation of Hindi words to their closely related languages. Our investigations show that TN performs the worst in recognising appropriate vowel translations for vowels used in Hindi words. While the outputs of HAN are the most reasonable ones after the post-processing step (described in Section 5.1) of removing repeating characters at the end of the word, the AM model performs moderately well in learning correct vowel translations. We attribute this to the fact that a Hindi word's vowels are mostly retained in its Bhojpuri cognate. The TN perhaps performs the worst at retaining vowels as it is infamous for being bad at copy mechanisms (Dehghani et al. 2018). These examples are shown in Table 7.

\subsubsection{Handling anusvāra}

An anusvāra is a diacritic used in a variety of written Indic scripts to denote a nasal sound, either a nasalized vowel or a nasal consonant that is not followed by a vowel. Anusvāra is used often in Hindi, but is absent in Bhojpuri writing. In Devanagari, the anusvāra is represented 
Table 7: Handling vowels

\begin{tabular}{c|cccc}
\hline Hindi & Bhojpuri (correct) & AM & HAN & TN \\
\hline आना & आइला & अयना & आना & अयन \\
[AnA] & [AilA] & [ayanA] & [AnA] & [ayana] \\
$\begin{array}{c}\text { (to come) } \\
\text { घुमाना }\end{array}$ & घुमावल & घुहावल & घुमावल & घोमावल \\
[GumAnA] & [GumaAvala] & [GuhAvala] & [GumaAvala] & [GomaAvala] \\
(to stir) & मउसी & मउसी & मउसी & मउसी \\
मौसी & [mausI] & [mausI] & [mausI] & [mausI] \\
[mOsI] & & & & \\
(maternal aunt) & & & &
\end{tabular}

by a dot (i) above a character, while it is represented by the letter ' $M$ ' in the WX notation. The two general patterns for translation of anusvāra are:

1. replacing them by adding an extra ' $F$ [na]' in front of the consonant, e.g. लेखों [leKoM] (writings)-> लेखन [leKana],

2. completely removing them, e.g. भेंट [BeMta] (offering)- $>$ भेट [Beta].

Our study shows that all three models get confused at choosing the correct rule and generally end up choosing the former one. Table 8 shows a few examples of this. Comparatively, the AM based model performs better than the other two.

6.1.4 Handling conjuncts for क्ष [kRa] and ज्ञ [jFa]

Conjuncts are formed when successive consonants, lacking a vowel in between them, physically join together. The conjuncts for क्ष [kRa] and ज्ञ $[\mathrm{jFa}]$ are special cases in that they are not clearly derived from the letters making up their components, i.e., the conjunct for क्ष [kRa] is क् $[\mathrm{k}]+$ ष [Ra] and for ज्ञ $[\mathrm{jFa}]$ it is ज् $[\mathrm{j}]+$ $[\mathrm{Fa}]$. The rules for translation of such conjuncts from Hindi to Bhojpuri are difficult to model (e.g. in the translation वृक्ष [vqkRa] (plant) to बिरिछ [biriCa], क्ष [kRa] becomes छ [Ca], while it remains as क्ष [kRa] in other cases); and therefore we explore the capability of the models in learning such translations. Our study shows that while the translation for क्ष [kRa] is easily learned by the models in most cases, the translation for ज्ञ [jFa] 
Table 8: Handling anusvāra

\begin{tabular}{|c|c|c|c|c|}
\hline Hindi & Bhojpuri (correct) & $\mathrm{AM}$ & HAN & $\mathrm{TN}$ \\
\hline कठिनाइयों & कठिनाइयन & कठिनाइयन & कठिनाइयन & कठिनाइन \\
\hline [kaTinAyioM] & [kaTinAyiana] & [kaTinAyiana] & [kaTinAyiana] & [kaTinAyiana] \\
\hline (hardships) & & & & \\
\hline लेखों & लेखन & लेखन & लेखन & लेखन \\
\hline [leKoM] & [leKana] & [leKana] & [leKana] & [leKana] \\
\hline (writings) & & & & \\
\hline भेंट & भेट & भेन्ट & भेन्स् & भेन्ट \\
\hline [BeMta] & [Beta] & [Benta] & [Ben] & [Benta] \\
\hline (offering) & & & & \\
\hline
\end{tabular}

Table 9: Handling conjuncts for क्ष [kRa] and ज्ञ [jFa]

\begin{tabular}{|c|c|c|c|c|}
\hline Hindi & Bhojpuri (correct) & AM & HAN & $\mathrm{TN}$ \\
\hline बरैक्षा & बरइक्षा & बरबक्धा & बरइक्षा & बरइक्षा \\
\hline [barEkRA] & [baraikRA] & [barabakDa] & [baraikRA] & [baraikRA] \\
\hline (village's name) & & & & \\
\hline अवैज्ञानिक & अबैज्ञानिक & अवैजज्ञानिक & अबैज्ञिानिक & अवैज्ञानिक \\
\hline [avEjFAnika] & [abEjFAnika] & [avEjajAnika] & [abEjianika] & [avEjFAnika] \\
\hline (unscientific) & & & & \\
\hline वृक्ष & बिरिछ & बक्छ् & बिक्षष & बिरिछ \\
\hline $\begin{array}{c}\text { [vqkRa] } \\
\text { (plant) }\end{array}$ & [biriCa & [bakC] & [bikRiRa] & [biriCa] \\
\hline
\end{tabular}

often results in ambiguity. Overall, TN performs the best in learning such translations while AM performs the worst. This, and many of the other errors mentioned in this section, are due to the nature of the writing system used. Table 9 shows a few examples of how different models handle conjuncts.

6.1.5 Handling the diacritic 'reph' for र् [r]: व [rva], वा [rvA], स्स्प [rspa]

र [r] in certain contexts is written as a special diacritic that takes the form of a curved upward dash above the preceding consonants. While translating the Hindi words containing such diacritics to their Bhojpuri counterparts, the diacritic is either replaced completely by र or simply 
NMT word transduction mechanisms for LRL

Table 10: Handling the diacritic for र् [a]

\begin{tabular}{|c|c|c|c|c|}
\hline Hindi & Bhojpuri (correct) & $\mathrm{AM}$ & HAN & $\mathrm{TN}$ \\
\hline अहिंसापूर्वक & अहिन्सापूर्वक & अहिन्सापूरन् & अहिन्सपूरसक & अहिन्साप्रवक \\
\hline $\begin{array}{c}\text { [ahiMsApUrvaka] } \\
\text { (nonviolently) }\end{array}$ & \multicolumn{4}{|c|}{ [ahinsApUrvaka] [ahinsApUrvan] [ahinsApUrasaka] [ahinsApravaka] } \\
\hline नाचपार्टी & नाचपारटी & नाचपार्ट & नाचपारप & नाचपारी \\
\hline [nAcapArtI] & [nAcpaAratI] & [nAcapArta] & [nAcapArapa] & [nAcapArI] \\
\hline (dance party) & & & & \\
\hline फर्नीचर & फरनीचर & फ्रीचर & फ्र & फरनीचर \\
\hline $\begin{array}{l}\text { [ParnIcara] } \\
\text { (furniture) }\end{array}$ & [ParanIcara] & [ParIcara] & [Pari] & [ParanIcara] \\
\hline
\end{tabular}

kept unchanged. Our results show that, in most cases, the AM model learns to preserve the diacritic as is; whereas the HAN and the TN models generally replace it by a complete $र$ [ra]. Table 10 shows a few examples of how different models handle these diacritics.

\subsubsection{Handling hrsva and deergha varna (short and long vowels)}

The Bhojpuri transliterations of most Hindi words show the same long and short vowels appearing after the corresponding consonants (words such as दूसरा [xUsarA] (second) can be treated as exceptions). We observe that for words preserving the nature of vowels (long or short), the AM and HAN based models perform better than that of the TN model while all the models fail to learn the cases where the nature of vowels (long or short) is switched upon translation. Interestingly, the TN model actually recognizes words in which the long vowel has to be switched to the short vowel and vice versa (we deduce this because it does not preserve the long/short nature in these cases) but it does not perform the switch correctly, and instead shows ambiguous behaviour on predictions. Table 11 shows a few examples of how different models handle short and long vowels.

6.2

6.2 .1

\section{Transduction-based properties}

$$
\text { Performance on long words }
$$

We consider words exceeding six characters in length to be long words. We found that while the translation quality of each model degrades as 
Saurav Jha, Akhilesh Sudhakar, Anil Kumar Singh

Table 11: Handling hrasva (short) and deergh (long) varna (letter)

\begin{tabular}{c|cccc}
\hline Hindi & Bhojpuri (correct) & AM & HAN & TN \\
\hline हिम [hima] (snow) & हिम [hima] & हिम [hima] & हिम [hima] & हीम [hIma] \\
दूसरा [dUsrA] (second) & दुसर [dusar] & दूसर [dUsar] & दूसर [dUsar] & दोसर [dosar] \\
घी [GI] (ghee) & घीव [GIva] & घी [GI] & घी [GI] & घि [GI] \\
\hline
\end{tabular}

Table 12: Performance on long words

\begin{tabular}{|c|c|c|c|c|}
\hline Hindi & Bhojpuri (correct) & AM & HAN & $\mathrm{TN}$ \\
\hline विद्यार्थियों & विद्यारथियन & बिद्यार्थयन & बिद्यारासियन & बिद्यारियन \\
\hline $\begin{array}{l}\text { [vidyArWiyoM] } \\
\text { (students) }\end{array}$ & [vidyAraWiyana] & [vidyArWayana] & [vidyArasiiyana] & [vidyAriyana] \\
\hline सुरक्षाकर्मीयों & सोरक्षाकरमियन & सुरक्जाकररनन & सुरक्षाक्रियन & सुरक्षारन \\
\hline $\begin{array}{c}\text { [surakRAkarmIyoM] } \\
\text { (guards) }\end{array}$ & [sorakRAkaramiyana & surakFAkararanana & [surakRAkairlyana & [surakRArana] \\
\hline हथियारबन्द & हथियारबन्द & हथियारबन्द & हथियारबन्द & हथियारब्द \\
\hline $\begin{array}{c}\text { [haWiyArabanda] } \\
\text { (armed) }\end{array}$ & [haWiyAraband] & [haWiyArabanda] & [haWiyArabanda] & [haWiyArabda] \\
\hline
\end{tabular}

word length increases, the AM based architecture is able to maintain the most sensible outputs, followed by the TN and the HAN based models. Table 12 shows examples of the behaviour of different models when they encounter long words.

\subsubsection{Identical transduction}

For words in Hindi that are written identically in Bhojpuri, we observe that the HAN based model gives the best results after post-processing (described in Section 5.1). The TN model fails in cases of longer words while the performance of the AM based model deteriorates for shorter words as well as vowels. Examples of behaviour in cases of identical transductions are presented in Table 13.

\subsubsection{Sensible yet erroneous translations}

We individually study the translations made by each model which sound legitimate when compared to the translations of other similar words but are actually wrong. For example, for diphthongs, while the 
NMT word transduction mechanisms for LRL

Table 13: Performance on identical translations

\begin{tabular}{|c|c|c|c|c|}
\hline Hindi & Bhojpuri (correct) & $\mathrm{AM}$ & HAN & $\mathrm{TN}$ \\
\hline मौलवी & मौलवी & मउबि & मउलवी & मउलवी \\
\hline $\begin{array}{c}\text { [mOlavI] } \\
\text { (Muslim doctor) }\end{array}$ & [mOlavI] & [maubi] & [maulavI] & [maulavI] \\
\hline झट & झट & झछट & झट & झट \\
\hline $\begin{array}{c}\text { [Jata] } \\
\text { (instant) }\end{array}$ & [Jata] & [JaCata] & [Jatatatata], [Jata] & [Jata] \\
\hline हथियारबन्द & हथियारबन्द & हथियारबन्द & हथियारबन्द & हथियारब्द \\
\hline $\begin{array}{c}\text { [haWiyArabanda] } \\
\text { (armed) }\end{array}$ & [haWiyArabanda] & [haWiyArabanda] & [haWiyArabanda] & [haWiyArabda] \\
\hline
\end{tabular}

Bhojpuri translation for भैया [BEyA] is भइया [BaiyA] (elder brother) (भै [BE] replaced by भइ [Bai]), the translation for कैमरा [kEmarA] (camera) does not follow such approach, whereby the character कै [kE] remains preserved. We observe that each model has its own types of erroneous translations due to such ambiguities as shown in Table 14.

Table 14: Some sensible yet erroneous translations

\begin{tabular}{r|ccc}
\hline Model & Hindi & Bhojpuri (correct) & Predicted \\
\hline AM & $\begin{array}{c}\text { तन्मयता [wanmayawA] } \\
\text { (concentration) }\end{array}$ & तन्मयता [wanmayawA] & तनमयता [wanamayawA] \\
कैमेरा [kEmerA] & कैमेरा [kEmerA] & कइमरा [kaimerA] \\
HAN & $\begin{array}{c}\text { (camera) } \\
\text { तरबूजा [warabUjA] } \\
\text { (watermelon) }\end{array}$ & तरबूजा [warabUjA] & तरबूज [warabUja] \\
यमुना [yamunA] & यमुना [yamunA] & जमुन [jamun] \\
(yamuna) & खिलाड़ी [KilAdI] & खेलारी [KelAdI] & खेलाड़ी [KelAdI] \\
(player) & उपकरण [upkaraNa] & उपकरन [upakaraNa] & ओपकरन [opkaraNa] \\
(equipment) & & \\
\hline
\end{tabular}




\subsubsection{Phonetically/orthographically invalid translations}

These are predicted transductions which do not follow the necessary phonetic rules in order to be pronounced. We study the type of such words individually for each model. Our findings suggest that the TN performs excellently in learning such rules since we did not notice any such instance of unpronounceable words present in the outputs of TN. Overall, HAN produced the most unpronounceable translations as can be seen from Table 15.

Table 15: Phonetically/orthographically invalid translations: for invalid predictions, a closest approximation of the WX notation is given

\begin{tabular}{|c|c|c|c|}
\hline Model & Hindi & Bhojpuri (correct) & Predicted \\
\hline \multirow[t]{3}{*}{$\mathrm{AM}$} & धर्मान्तरण & धरमान्तरन & धर्मान्थि \\
\hline & [XarmanwaraNa] & [Xaramanwarana] & [Xarmaani] \\
\hline & (religious conversion) & & \\
\hline \multirow[t]{3}{*}{$\mathrm{AM}$} & सुरक्षाकर्मीयों & सोरक्षाकरमियन & सुरक्षाक्रियन \\
\hline & [surakRAkarmIyoM] & [surakRAkaramIyana] & [surakRAkairIyana] \\
\hline & (security guards) & & \\
\hline \multirow[t]{2}{*}{$\mathrm{AM}$} & वर्जित & वरजित & बरामि \\
\hline & [varjiwa] (contraband) & [varajiwa] & [baraimi] \\
\hline \multirow[t]{2}{*}{ HAN } & कुंजी & कुन्जी & कुी \\
\hline & [kuMjI] (key) & [kunjI] & [kuMI] \\
\hline \multirow[t]{2}{*}{ HAN } & अवैज्ञानिक & अबैज्ञानिक & अवैजज्ञानिक \\
\hline & [avEjFAnika] (unscientific) & [abEjFAnika] & [avEjajAnika] \\
\hline
\end{tabular}

6.3

Limitations of the method

While training the system on cognate pairs ensures that the model does not require exhaustive resources such as a parallel corpora, the fact remains that cognate-property is inherently confined to the case of closely-related languages. As described in Section 1, Bhojpuri is closely related to Hindi; it is a dialect/immediate sibling and shares the same script. ${ }^{13}$ By contrast, Bangla (Section 5.3) is a direct descendant of

${ }^{13}$ Although Bhojpuri was historically written in Kaithi scripts, those have become obsolete; Devanagari is now the primary script. 
Sanskrit-family or Pali ${ }^{14}$ to Prakrit and Apabhramsha (Chatterji 1926; Bali 2016) but is only loosely related to Hindi: it is neither a dialect nor an immediate sibling of Hindi; it has its own script; and it has word formation rules that are sometimes contrary to Hindi. The effects of such increased 'language distance' are apparent from Table 4 (HindiBhojpuri) and Table 5 (Hindi-Bangla) where Hindi-Bhojpuri cognate pairs show far better transduction quality than Hindi-Bangla.

Further, with the increased distance between the source and the target language in a language pair, it is safe to say that the set of cognates shared by these dwindles (Beel and Felder 2014), since cognates, by definition, are word pairs that not only have similar meaning and phonetics but also reflect an allied linguistic derivation. We observed this even in our own cognate datasets: the Hindi-Bangla dataset had 25\% fewer cognates than the Hindi-Bhojpuri dataset (see Section 5.3). Having fewer real-world cognate pairs means that even if a nominally complete dataset of all cognates were to be compiled, there would still be comparatively less data to train models on, hence exhibiting another limitation in extending the method to language pairs of arbitrary distance.

\section{IMPROVEMENTS ON HINDI - BHOJPURI MAGHINE TRANSLATION}

This section talks of the improvements we make on machine translation from Hindi to Bhojpuri. The authors would like to mention two key points here. First, this section stands distinguished from the rest of the paper in that the methods and results discussed thus far hold for word-to-word transduction. In contrast, we now depict how such transductions can be used to improve the accuracy of a machine translation system. Second, we must mention that to the best of our knowledge, no prior machine translation systems have been trained on a Hindi-Bhojpuri parallel corpus of a trainable size, simply because such a corpus does not exist.

\footnotetext{
${ }^{14}$ Hindi descended from Prakrit, Apabhramsha and Perso-Arabic while Bangla and Bhojpuri inherited the regional dialects of Apabhramsha. Consequently, Bangla and Bhojpuri, sharing several regional boundaries of Northern India, Bangladesh and Nepal, can be thought of having similar ancestral languages to Hindi.
} 
For our purposes, we build an artifical parallel corpus in the following manner. We first scrape four Bhojpuri blogs for Bhojpuri sentences: Anjoria, ${ }^{15}$ TatkaKhabar, ${ }^{16}$ Bhojpuri Manthan, ${ }^{17}$ and Bhojpuri Sahitya Sarita. ${ }^{18}$ From these, we collect a set of approximately 40,000 Bhojpuri sentences. We then follow a two-step process for obtaining Hindi translations of these sentences using the Google Translate API. Since the API does not offer Bhojpuri as a source language, we first use the Hindi-English API for translating the Bhojpuri sentences to English (as an intermediate language). We then translate these English sentences to Hindi using the English-Hindi API. Although this process gives us noisy translations between Bhojpuri and Hindi, this method is based upon the hypotheses that:

1. in the absence of an actual reported machine translation system between these languages, back-translation can be used as the only available substitute for showing improvement due to word transduction;

2. that this is the best available solution in the absence of HindiBhojpuri parallel corpora, let alone a pre-trained Hindi-Bhojpuri translation system; and

3. that Bhojpuri is sufficiently similar to Hindi that its linguistic properties remain preserved satisfactorily throughout the intermediate processing.

Also, the choice of English as an intermediate language is based on the fact that it gives the best BLEU scores when Hindi is fixed as the other language in the translation pair. At the time of writing this work, there is no Bhojpuri-Hindi API (or publicly reported Hindi-Bhojpuri machine translation system), while trivially translating using the HindiHindi API does not seem to work as the API simply copies the inputs to outputs. For reporting the machine translation results, we use the standard document-level BLEU score as suggested by Papineni et al. (2002).

For the held-out test set, we curated 1000 sentences for which ground truth translations were manually obtained from experts, and

\footnotetext{
15 https://www.anjoria.com/

16 http://khabar .anjoria.com/

17 http://bhojpurimanthan.com/

18 http://www. bhojpurisahityasarita.com/
} 
NMT word transduction mechanisms for LRL

\begin{tabular}{r|cr}
\hline & Training set & Test set \\
\hline Number of sentences & 40,000 & 1,000 \\
Total number of tokens & 812,070 & 19,689 \\
Number of unique tokens & 20,551 & 620 \\
\hline
\end{tabular}

Table 16:

Machine translation corpus statistics

not artificially generated. Statistics on training and test sentences can be found in Table 16.

We train a Bi-LSTM based encoder-decoder network (500 units) with Luong attention, as described in Luong et al. (2015a) (we use OpenNMT's global attention implementation) on the training set for Hindi-Bhojpuri translation. The model thus trained resulted in a BLEU score of around 7.1 on the test set, which is not surprising given the method of training. With this as the baseline, we make corrections to this model's output. We first align each Hindi sentence and its Bhojpuri translation, by aligning pairs of source-target words based on their pairwise weights in the attention matrix. Following this, we identify OOV Hindi words using a simple dictionary-based approach as has been suggested by Bahdanau et al. (2014). We use a shortlist of 15,000 most common words in Hindi, obtained from the Hindi Wikipedia monolingual corpus, and treat all other words as OOV. We experiment with 5000, 10,000, 15,000, and 20,000 most common words, and find the 15,000 word shortlist produced the best BLEU score improvements. We then replace the translation (as obtained by the alignments using the attention matrix) of each OOV word with its corresponding transduction generated by our word transduction model. Replacing the translation of OOV words with that of their transductions leads to an improvement of 6.3 points in the BLEU score, which is substantial considering that we are translating to a low-resource language. We obtain a BLEU score of 13.4 with such a basic translation set-up followed by simple correction of OOV translations using transductions.

More importantly, the improvement in the MT BLEU score shows that even though the task of transduction is a focused one, it generalizes well to OOV Hindi words that are not part of a Hindi-Bhojpuri cognate pair. This stands as an important aspect of our work. Our transduction model, despite being trained on a dataset that is constrained to cognate pairs, lends reasonable improvements to the highly 
generalized machine translation task by exploiting the closeness of Hindi and Bhojpuri.

\section{GONGLUSION}

We propose a character-level transduction of OOV words between a pair of closely related languages, out of which at least one is a low-resource language. Word transduction aims to predict the orthographic form of the word in the target language, given the word in the source language. We restrict the training space to a set of cognates, since in the case of closely-related languages, a cognate can be a good approximation to a translation, if not the translation itself. We present three different models for the same, each of which performs well on handling certain types of grapheme transformations, while performing sub-optimally on others. While all our models ${ }^{19}$ outperform the current state of art for Hindi-Bhojpuri transduction, the TN model gives the overall best performance. We suggest a two-step procedure to improve a low-resource NMT system by:

1. identifying the need to handle OOV words separately; and

2. transducing them to their target equivalents, instead of translating.

In the process, we also propose a primitive yet useful MT method using Google Translate APIs for a pair of languages which has no known MT system. In the future, we would like to test our models on more closely related language pairs. Further, we would like to build a stateof-the-art MT pipeline for low resource languages, which incorporates our method to handle OOV words. We would also like to compare the effect of transduction as a solution to the OOV problem faced by wordlevel MT systems by comparing it to a character-level MT system.

\section{REFERENGES}

Martín Abadi, Ashish Agarwal, Paul Barham, Eugene Brevdo, Zhifeng Chen, Craig Citro, Greg S. Corrado, Andy DAvis, Jeffrey DeAn, Matthieu Devin, Sanjay GHemaWAT, Ian Goodfellow, Andrew HaRP, Geoffrey

\footnotetext{
${ }^{19}$ We open source the code and data curated for this work in our github repo: https://github.com/Saurav@๑74/nmt - based-word-transduction.
} 
IRVING, Michael ISARD, Yangqing JIA, Rafal JozEFowICZ, Lukasz KAISER, Manjunath Kudlur, Josh Levenberg, Dan MANÉ, Rajat MongA, Sherry MoOre, Derek Murray, Chris Olah, Mike SCHuSter, Jonathon SHLENS, Benoit Steiner, Ilya SutSkeVer, Kunal TAlWAR, Paul Tucker, Vincent VANHoucke, Vijay VAsudeVAn, Fernanda ViÉgas, Oriol Vinyals, Pete WARden, Martin Wattenberg, Martin Wicke, Yuan Yu, and Xiaoqiang ZHENG (2015), TensorFlow: Large-Scale Machine Learning on Heterogeneous Systems, https://www. tensorflow.org/, software available from tensorflow.org.

Ankita ACHARYA (2015), Contrastive Study of Bundeli and Hindi Pronunciation, in regICON-2015: Regional Symposium on Natural Language Processing, Varanasi, India.

Yaser AL-OnAIZAN, Jan CuRIN, Michael JAHR, Kevin KNIGHT, John LAFFERTy, Dan MElAmed, Franz-Josef OCH, David PuRDy, Noah A. SMITH, and David YAROWSKY (1999), Statistical Machine Translation, in Final Report, JHU Summer Workshop, volume 30.

Jimmy BA, Ryan KIROs, and Geoffrey E. HINTON (2016), Layer Normalization, Computing Research Repository (CoRR), abs/1607.06450.

Dzmitry BAHDANAU, Kyunghyun CHO, and Yoshua Bengio (2014), Neural Machine Translation by Jointly Learning to Align and Translate, Computing Research Repository (CoRR), abs/1409.0473.

Purnima BALI (2016), Evolution of Bengali Literature: An Overview, International Journal of English Language, Literature and Translation Studies, 3:325-332.

Rachel BEEL and Jennifer N. FELDER (2014), Phonological Adaptations of English Loanwords in Turkish, in Proceedings of the Big South Undergraduate Research Symposium (BigSURS), High Point, NC, USA.

Yoshua Bengio, Patrice Y. SimARD, and Paolo FrAsConi (1994), Learning long-term dependencies with gradient descent is difficult, IEEE Transactions on Neural Networks, 5(2):157-66.

Luisa Bentivogli, Arianna Bisazza, Mauro Cettolo, and Marcello FEDERICO (2016), Neural versus Phrase-Based Machine Translation Quality: a Case Study, in Proceedings of the Conference on Empirical Methods in Natural Language Processing (EMNLP), pp. 257-267, Austin, TX, USA.

Piotr Bojanowski, Edouard Grave, Armand Joulin, and Tomas Mikolov (2017), Enriching Word Vectors with Subword Information, Transactions of the Association for Computational Linguistics (TACL), 5:135-146.

Suniti Kumar CHATTERJI (1926), The Origin and Development of the Bengali Language, Calcutta University Press. 
Aditi Chaudhary, Chunting Zhou, Lori S. Levin, Graham Neubig, David R. Mortensen, and Jaime G. CARBonell (2018), Adapting Word Embeddings to New Languages with Morphological and Phonological Subword Representations, in Proceedings of Conference on Empirical Methods in Natural Language Processing, pp. 3285-3295, Brussels, Belgium.

Qiming CHEN and Ren WU (2017), CNN Is All You Need, Computing Research Repository (CoRR), abs/1712.09662.

David ChIANG (2005), A Hierarchical Phrase-based Model for Statistical Machine Translation, in Proceedings of the 43rd Annual Meeting on Association for Computational Linguistics, pp. 263-270, Association for Computational Linguistics, Ann Arbor, Michigan.

Kyunghyun Cho, Bart VAN MERRIËNBOER, Caglar GulCEHRE, Dzmitry BAHDANAU, Fethi Bougares, Holger SchWEnK, and Yoshua Bengio (2014), Learning phrase representations using RNN encoder-decoder for statistical machine translation, in Proceedings of the 2014 Conference on Empirical Methods in Natural Language Processing (EMNLP), pp. 1724--1734, Doha, Qatar.

François CHOLLET et al. (2015), Keras, https://github.com/keras - team/keras.

Monojit CHOUdHuRY (2003), Rule-based grapheme to phoneme mapping for Hindi speech synthesis, in 90th Indian Science Congress of the International Speech Communication Association (ISCA), Bangalore, India.

Mostafa Dehghani, Stephan Gouws, Oriol Vinyals, Jakob UszKoreit, and Lukasz KAISER (2018), Universal Transformers, Computing Research Repository (CoRR), abs/1807.03819.

Etienne DENOUAL and Yves LEPAGE (2005), BLEU in characters: towards automatic MT evaluation in languages without word delimiters, in Companion Volume to the Proceedings of the Second International Joint Conference on Natural Language Processing, pp. 81-86.

Etienne DENOUAL and Yves LEPAGE (2006), The character as an appropriate unit of processing for non-segmenting languages, in Speech Processing Society 12th Annual Meeting (NLP2006), pp. 731-734, Hiroshima University, Japan.

Inger EKMAN and Kalervo JÄRVELIN (2007), Spoken Document Retrieval in a Highly Inflectional Language, in Proceedings of the NODALIDA Conference, pp. 44-50.

Andrew FinCH and Eiichiro Sumita (2009), Transliteration by bidirectional statistical machine translation, in Proceedings of the 2009 Named Entities Workshop: Shared Task on Transliteration, Joint conference of the 47th Annual Meeting of the Association for Computational Linguistics and the 4th International Joint Conference on Natural Language Processing of the Asian Federation of Natural Language Processing, pp. 52-56, Association for Computational Linguistics (ACL), Singapore. 
Mikel L. FORCADA and Ramón P. ÑECo (1997), Recursive Hetero-associative Memories for Translation, in IWANN.

Sir George GRIERSON (1928), Linguistic Survey of India, The Journal of the Royal Asiatic Society of Great Britain and Ireland, 3:711-718.

Rohit GuPTA, Pulkit Goyal, and Sapan DiWAKar (2010), Transliteration among Indian Languages using WX Notation, in Proceedings of KONVENS, pp. 147-150, Saarbrücken, Germany.

Jan HAJIČ (2000), Machine Translation of Very Close Languages, in Proceedings of the Applied Natural Language Processing Conference (ANLP), pp. 7-12, Seattle, Washington, USA.

Kaiming HE, Xiangyu ZHANG, Shaoqing REN, and Jian Sun (2016), Deep Residual Learning for Image Recognition, Proceedings of the IEEE Conference on Computer Vision and Pattern Recognition (CVPR), pp. 770-778.

Sébastien JeAn, Kyunghyun CHo, Roland MEMisevic, and Yoshua BENGio (2015), On Using Very Large Target Vocabulary for Neural Machine Translation, in Joint conference of the 47th Annual Meeting of the Association for Computational Linguistics and the 4th International Joint Conference on Natural Language Processing of the Asian Federation of Natural Language Processing, pp. 1-10, Beijing, China.

Nal KALChbRenner and Phil Blunsom (2013), Recurrent Continuous Translation Models, in Proceedings of the Conference on Empirical Methods in Natural Language Processing (EMNLP), pp. 1700-1709, Seattle, USA.

Grzegorz KondRAK, Daniel MARCU, and Kevin KNIGHT (2003), Cognates can improve statistical translation models, in Proceedings of the 2003 Conference of the North American Chapter of the Association for Computational Linguistics on Human Language Technology: companion volume of the Proceedings of HLT-NAACL 2003-short papers-Volume 2, pp. 46-48, Association for Computational Linguistics, Edmonton, Canada.

Siwei LAI, Liheng XU, Kang LIU, and Jun ZHAO (2015), Recurrent Convolutional Neural Networks for Text Classification, in Proceedings of the Association for the Advancement of Artificial Intelligence (AAAI), Austin, TX, USA. Naihan LI, Shujie LIU, Yanqing LIU, Sheng ZHAO, Ming LIU, and Ming ZHOU (2018), Close to human quality TTS with transformer, arXiv preprint arXiv:1809.08895.

Minh-Thang LuONG and Christopher D. MANNING (2016), Achieving Open Vocabulary Neural Machine Translation with Hybrid Word-Character Models, Computing Research Repository (CoRR), abs/1604.00788.

Thang LuONG, Hieu PHAM, and Christopher D. MANNING (2015a), Effective Approaches to Attention-based Neural Machine Translation, in Proceedings of the Conference on Empirical Methods in Natural Language Processing (EMNLP), pp. 1412-1421, Lisbon, Portugal. 
Thang LuONG, Ilya SutskeVER, Quoc V. Le, Oriol Vinyals, and Wojciech ZAREMBA (2015b), Addressing the Rare Word Problem in Neural Machine Translation, in Joint conference of the 47th Annual Meeting of the Association for Computational Linguistics and the 4th International Joint Conference on Natural Language Processing of the Asian Federation of Natural Language Processing, pp. 11-19, Beijing, China.

B.P. Mahapatra, P. PAdManabha, G.D. McConnell, and V.S. Verma (1989), The Written Languages of the World: A Survey of the Degree and Modes of Use: Volume 2: India: Book 1 Constitutional Languages, volume 1, Quebec : Presses de l'Université Laval; Forest Grove, Oregon.

Gideon S MANN and David YAROWSKY (2001), Multipath translation lexicon induction via bridge languages, in Proceedings of the Second Meeting of the North American Chapter of the Association for Computational Linguistics on Language technologies, pp. 1-8, Association for Computational Linguistics, Pittsburgh, PA, USA.

Tomas Mikolov, Ilya Sutskever, Kai Chen, Greg S. CorRado, and Jeff DEAN (2013), Distributed representations of words and phrases and their compositionality, in Advances in Neural Information Processing Systems (NIPS), pp. 3111-3119.

Diwakar MishrA and Kalika BALI (2011), A Comparative Phonological Study of the Dialects of Hindi, in Proceedings of the International Congress of Phonetic Sciences (ICPhS), pp. 1390-1393, Hong Kong.

Preslav NAKov and Jörg TIEDEMANN (2012), Combining word-level and character-level models for machine translation between closely-related languages, in Proceedings of the 50th Annual Meeting of the Association for Computational Linguistics: Short Papers-Volume 2, pp. 301-305, Association for Computational Linguistics (ACL), Jeju Island, Korea.

Kishore PAPINENI, Salim Roukos, Todd WARD, and Wei-Jing ZHU (2002), BLEU: a method for automatic evaluation of machine translation, in Proceedings of the 40th Annual Meeting on Association for Computational Linguistics, pp. 311-318, Association for Computational Linguistics, Pennsylvania, PA, USA.

Jeffrey Pennington, Richard Socher, and Christopher MANNING (2014), Glove: Global vectors for word representation, in Proceedings of the 2014 Conference on Empirical Methods in Natural Language Processing (EMNLP), pp. 1532-1543, Doha, Qatar.

Ngoc-Quan PhAm, Jan NieHues, and Alexander H. WAIBEL (2018), Towards one-shot learning for rare-word translation with external experts, in Proceedings of the 2nd Workshop on Neural Machine Translation and Generation, 56th Annual Meeting of the Association for Computational Linguistics, pp. 100-109, Melbourne, Australia. 
Martin POPEL and Ondřej BOJAR (2018), Training Tips for the Transformer Model, The Prague Bulletin of Mathematical Linguistics, 110(1):43-70.

Maja Popović (2015), chrF: character n-gram F-score for automatic MT evaluation, in Proceedings of the Tenth Workshop on Statistical Machine Translation, pp. 392-395, Lisbon, Portugal.

Kevin P. SCANNELL (2006), Machine translation for closely related language pairs, in Proceedings of the Workshop Strategies for Developing Machine Translation for Minority Languages, pp. 103-109, Citeseer, Genoa, Italy.

Rico SENNRICH, Barry HADDOW, and Alexandra BIRCH (2016), Neural Machine Translation of Rare Words with Subword Units, Computing Research Repository (CoRR), abs/1508.07909.

Shashikant SHARMA and Anil Kumar SINGH (2017), Word Transduction for Addressing the OOV Problem in Machine Translation for Similar

Resource-Scarce Languages, in Proceedings of the International Conference on Finite State Methods and Natural Language Processing (FSMNLP), pp. 56-63, Umeå, Sweden.

Michel SimARD, George F. Foster, and Pierre ISABELLE (1993), Using cognates to align sentences in bilingual corpora, in Proceedings of the 1993 Conference of the Centre for Advanced Studies on Collaborative Research: Distributed Computing - Volume 2, pp. 1071-1082, IBM Press.

Smriti SINGH and Vaijayanthi M. SARMA (2010), Hindi noun inflection and Distributed Morphology, in Proceedings of the International Conference on Head-Driven Phrase Structure Grammar, pp. 307-321.

Srishti SINGH and Girish Nath JHA (2015), Statistical Tagger for Bhojpuri (employing Support Vector Machine), in Proceedings of the International Conference on Advances in Computing, Communications and Informatics (ICACCI), pp. 1524-1529, Kochi, India.

Martin SUNDERMEYER, Ralf SCHLÜTER, and Hermann NEY (2012), LSTM neural networks for language modeling, in Proceedings of Thirteenth Annual Conference of the International Speech Communication Association, pp. 194-197, Portland, OR, USA.

Ilya SutSKEVER, Oriol VINYALS, and Quoc V. LE (2014a), Sequence to Sequence Learning with Neural Networks, in Advances in neural information processing systems (NIPS).

Ilya SuTSKEVER, Oriol VINYALS, and Quoc V Le (2014b), Sequence to sequence learning with neural networks, in Advances in Neural Information Processing Systems (NIPS), pp. 3104-3112.

Jörg TiEdEMANN (2012), Character-based pivot translation for under-resourced languages and domains, in Proceedings of the 13th Conference of the European Chapter of the Association for Computational Linguistics, pp. 141-151, Association for Computational Linguistics, Avignon, France. 
Robert L. TRAmmell (1971), The Phonology of the Northern Standard Dialect of Bhojpuri, Anthropological Linguistics, 13(4):126-141.

Zhaopeng TU, Yang LIU, Lifeng SHANG, Xiaohua LIU, and Hang Li (2017), Neural Machine Translation with Reconstruction, in Association for the Advancement of Artificial Intelligence (AAAI), San Francisco, CA, USA.

Ashish VASWANI, Noam SHAZEer, Niki PARMAR, Jakob UszKoreIt, Llion Jones, Aidan N. Gomez, Lukasz KAISER, and Illia PolosukHIN (2017), Attention is All you Need, in Advances in Neural Information Processing Systems (NIPS).

David Vilar, Jan-T. Peter, and Hermann Ney (2007), Can We Translate Letters?, in Proceedings of the Second Workshop on Statistical Machine Translation, StatMT '07, pp. 33-39, Association for Computational Linguistics, Stroudsburg, PA, USA, http: //dl . acm . org/citation . cfm?id=1626355.1626360.

Oriol Vinyals, Łukasz KaISER, Terry Koo, Slav Petrov, Ilya SutSKeVER, and Geoffrey E. HINTON (2015), Grammar as a Foreign Language, in Advances in neural information processing systems (NIPS).

Yonghui Wu, Mike SCHUSTER, Zhifeng CHEN, Quoc V LE, Mohammad NorouzI, Wolfgang MACHEREY, Maxim Krikun, Yuan CAO, Qin GAO, Klaus MACHEREY, et al. (2016), Google's Neural machine translation system: Bridging the gap between human and machine translation, Computing Research Repository (CoRR), abs/1609.08144.

Zichao YANG, Diyi YANG, Chris Dyer, Xiaodong He, Alexander J. SMOlA, and Eduard H. Hovy (2016), Hierarchical Attention Networks for Document Classification, in Proceedings of the Annual Conference of the North American Chapter of the Association for Computational Linguistics (HLT-NAACL), pp. 1480-1489, San Diego, CA, USA.

This work is licensed under the Creative Commons Attribution 3.0 Unported License. http://creativecommons.org/licenses/by/3.๑/

(c) BY 